\title{
Pediatric Anesthesia
}

ORIGINAL ARTICLE

\section{A comparison of the postoperative pain experience in children with and without attention-deficit hyperactivity disorder (ADHD)}

\author{
Sondra Rosander ${ }^{1}$, Rebecca Nause-Osthoff ${ }^{1}$, Terri Voepel-Lewis ${ }^{1}$ \& Alan R. Tait ${ }^{1,2}$ \\ 1 Division of Pediatric Anesthesiology, University of Michigan Health System, Ann Arbor, MI, USA \\ 2 Center for Behavioral and Decision Sciences in Medicine, University of Michigan Health System, Ann Arbor, MI, USA
}

\section{What is already known}

- Children with ADHD have the potential for deterioration in ADHD symptoms perioperatively.

- Physiologic evidence and imaging studies suggest differences in the brains of those with ADHD, which could lead to differences in how pain is processed.

\section{What is new}

- Compared with children without ADHD there was no difference in perioperative pain experience in children with ADHD.

- Children with ADHD experienced an increased time to return to normal activity compared with those without ADHD.

\section{Implications for translation}

- Patients and families may be counseled that children with ADHD may take longer to return to baseline activity following surgery and anesthesia than those without ADHD.

\section{Keywords}

attention-deficit hyperactivity disorder; child; postoperative pain

\section{Correspondence}

Sondra Rosander, Division of Pediatric Anesthesiology, 4-911 Mott Children's Hospital, 1540 E. Hospital Drive, SPC 4245, Ann Arbor, MI 48109-4245, USA

Email: buists@umich.edu

Section Editor: Adrian Bosenberg

Accepted 30 May 2015

doi:10.1111/pan.12720

\section{Summary}

Background: Children with attention-deficit hyperactivity disorder (ADHD) may experience pain differently compared to other children, yet the evidence is equivocal regarding whether pain is heightened or dampened. This prospective observational study, therefore, was designed to compare the postoperative pain experiences in children with and without ADHD.

Methods: Children aged 7-17 years with a diagnosis of ADHD $(n=119)$ who were scheduled for a surgical procedure requiring postoperative pain management and a matched cohort of children without ADHD were recruited $(n=122)$. Postoperative pain scores and analgesic use were recorded for 1 week, as was parents' estimate of their child's return to normal activity.

Results: There were no differences in highest pain scores between children with ADHD (3.3 $\pm 2.5,0-10$ numerical rating scale) and those without $(2.8 \pm 1.9)$. Postoperative opioid use was also similar on day 1 following surgery $\left(0.12 \pm 0.3 \mathrm{mg} \cdot \mathrm{kg}^{-1}\right.$ vs $0.08 \mathrm{mg} \cdot \mathrm{kg}^{-1} \pm 0.1$ morphine equivalents, respectively). Children with $\mathrm{ADHD}$, however, had a significantly longer return to normal activity $(4.9 \pm 3.8$ vs $3.8 \pm 3.0$ days; $P<0.05)$.

Conclusions: Results suggest that there were no differences in the postoperative pain experiences of children with and without ADHD. However, the 
observation that children with ADHD took longer to return to baseline activity will be important in educating parents regarding their child's postoperative experience.

\section{Introduction}

The estimated prevalence of attention-deficit hyperactivity disorder (ADHD) in children is $5.29 \%$ worldwide (1), and thus presents as a co-morbid symptom for many children undergoing general anesthesia. Recent data suggest the potential for perioperative deterioration of ADHD symptoms including behavioral and sleep disturbances (2) and increased maladaptive behaviors postoperatively in these children (3), yet it remains unknown whether pain or other perioperative factors contribute to these and other outcomes. Observed and postulated physiologic mechanisms suggest that children with ADHD may experience pain differently than their nonADHD counterparts. For instance, reported differences in somatosensory responses $(4,5)$, locus coeruleus function (6-8), and hypothalamic-pituitary-adrenal axis (HPA) hypoactivity $(9,10)$ in persons with ADHD suggest the potential for enhanced pain response. Conversely, structural and functional magnetic resonance imaging scans in persons with ADHD suggest the potential for blunted pain perception $(11,12)$.

Given the importance of pain management during and following anesthesia, a better understanding of whether and how the pain experience differs for children with ADHD is warranted. We, therefore, conducted a prospective, observational study to explore potential differences in the pain and analgesic response of children with ADHD compared with those without this condition. We hypothesized that there would be a difference in self-reported pain intensity scores in children aged $7-$ 17 years old with ADHD compared with children without ADHD. Secondary aims were to determine if children with and without ADHD differ with respect to opioid and nonopioid medications administered, postoperative nausea and vomiting, and days to return to normal activity.

\section{Materials and methods}

Following Institutional Review Board approval, we conducted a prospective matched cohort observational study in children with and without ADHD undergoing painful outpatient surgical procedures at the University of Michigan C.S. Mott Children's Hospital from January 2010 to August 2013. Enrollment into this study followed written parental consent and verbal child assent on the morning of surgery.
All children between the ages of 7-17 years old undergoing an outpatient or overnight stay surgical procedure associated with pain were eligible. Children with ADHD were identified preoperatively based on the presence of this diagnosis or a prescription for stimulant medication, and as confirmed by interview with the parent prior to surgery. Children were excluded from this study if they had a history of chronic pain or analgesic use. Case-matched controls were recruited from the remaining cohort of children without ADHD based on an a priori procedure-, age-, and gender-matched selection process.

Postoperatively, pain was assessed on awakening and every 15 min until postanesthesia care unit (PACU) discharge using the 0-10 Numerical Rating Scale (NRS) which is a widely utilized valid and reliable scale for this age range $(13,14)$. Prior to discharge from the hospital, parents were given a worksheet to assess the child's global pain intensity score per parent and child report prior to any analgesic pain medication administration at home. The number and amounts of opioids and nonopioids administered in the $24 \mathrm{~h}$ following PACU discharge were recorded. Parents were contacted via telephone at 7 days after discharge and interviewed to record the data documented on the worksheet, the current global pain intensity score and overall comfort level of the child, as well as the parent's global perception of the day that the child returned to his or her normal activity.

\section{Statistical analysis}

Data analysis was carried out using SPSS ${ }^{\circledR}$ statistical software (version 19, IBM Corp., Kingston, NY, USA). Descriptive statistics were used to compare the demographics of the ADHD group and the matched cohort groups. The outcomes of interest were compared between the two groups using the two-tailed $t$-test for outcomes with continuous variables (e.g., morphine equivalents) and chi-squared test for outcomes with categorical values (e.g., ASA I/II). The nonparametric analysis of variance (Kruskal-Wallace) test with repeated measures was performed for comparison of the NRS pain intensity scores between the two groups. The significance levels were set at 0.05 and inference testing was two-tailed.

The necessary sample size was calculated to detect a 1-point difference on the NRS, which has been found to be clinically meaningful (i.e., pain relief or worsening) 
for children in this age range (14). Given that the average postoperative pain score in our general outpatient surgical population was determined to be $4.68 \pm 2.8$, we required 240 children (120 per group) to detect a 1-point difference in pain intensity scores between groups ( $\alpha=0.05, \beta=0.20$, two-sided).

\section{Results}

A total of 261 children were approached to participate of which 18 declined and 2 were deemed ineligible due to a lengthy inpatient stay. Complete data were thus available for 241 subjects (119 children with ADHD and 122 controls without ADHD). Subjects were well-matched for age, gender, surgical procedure, and invasiveness (15), with the exception of ASA status and previous surgical experience (Table 1). In addition, use of regional or local anesthetics and premedication with midazolam was similar between the groups (Table 1). Most of the children in the ADHD group (78\%) were taking medications for their disorder at the time of surgery (last dose within 1 week), and the median time from last dose prior to surgery was -1.0 day (interquartile range -1.24 to -0.22 ), which likely reflects withholding this

Table 1 Demographics

\begin{tabular}{lcc}
\hline & No ADHD, & ADHD, \\
$n=122$ & $n=119$ \\
\hline Age (yrs) & $12.5 \pm 3.0$ & $12.4 \pm 3.3$ \\
ASA (I/II, \%) & $60.7 / 39.3$ & $32.8 / 67.2$ \\
Gender (M/F, \%) & $71.3 / 28.7$ & $72.3 / 27.7$ \\
Race/ethnicity & & \\
White & $100(82.0)$ & $99(83.2)$ \\
Black & $12(9.8)$ & $15(12.6)$ \\
Hispanic & $0(0.0)$ & $3(2.5)$ \\
Other & $10(8.2)$ & $2(1.7)$ \\
Previous surgery (Yes) & $62(50.8)$ & $83(69.7)$ \\
Surgical severity score A & $62(50.8)$ & $67(56.3)$ \\
Surgical service & & \\
Otolaryngology & $23(18.9)$ & $24(20.2)$ \\
Orthopedics & $37(30.3)$ & $39(32.8)$ \\
General surgery & $19(15.6)$ & $15(12.6)$ \\
Plastic surgery & $10(8.2)$ & $11(9.2)$ \\
Urology & $14(11.5)$ & $14(11.8)$ \\
Ophthalmology & $10(8.2)$ & $9(7.6)$ \\
Other & $9(7.4)$ & $7(5.9)$ \\
Use of local/ & $62(51)$ & $68(57)$ \\
regional anesthetic & & $65(54.6)$ \\
Midazolam & $69(57)$ & \\
premedication & & \\
\hline
\end{tabular}

Data are described as mean \pm SD and $n(\%)$. ADHD, attention-deficit hyperactivity disorder; ASA, American society of anesthesiologists physical status; Surgical severity score $A=$ minimally invasive surgery (15). type of medication after the child is made nil per os (NPO), per general institutional guidelines. We have found that some parents routinely withhold these medications during school breaks, including weekends.

There were no statistically significant differences in any measure of the perioperative pain experience (pain scores and perioperative opioid requirements) between the groups (Table 2). The majority of children in both the groups were treated for pain at home after discharge (86\% of the ADHD group vs $85 \%$ of the controls, $P=0.77)$. There were no differences between the groups in parent or child report of pain intensity after discharge or in day 1 analgesic use. However, there was a statistically significant increase in the number of days to return to normal activity for the children with ADHD (4.88 vs 3.78 days, mean difference $-1.1, \mathrm{CI}-1.92,-0.29$ ).

To examine whether the pain experiences differed between the subgroups of children currently managed with medications for ADHD $(n=91)$ and those not on medications or those without ADHD, we examined pain scores, medication use, and recovery parameters for these groups using a one-way analysis of variance, with Bonferroni corrections for multiple comparisons. Pain score and opioid use data for these groups are shown in Table 3. Although pain scores and opioid use on day 1 were slightly higher for the children being medically managed for their ADHD, these differences did not achieve statistical significance $[F$ statistic for overall pain ratings postdischarge $=2.06(\mathrm{df} 2), P=0.13]$.

\section{Discussion}

This study is the first to our knowledge to examine whether children with ADHD differ in their perioperative pain experience compared with children without ADHD. Results showed no statistical difference in the perioperative pain experience in children with and without ADHD in this sample. However, children with ADHD had a longer time to return to normal activity based on parent global assessment of the child. These findings are important as we develop anesthetic plans and counsel patients, parents, and families during the perioperative period.

The previously existing data regarding the pain experience in children with ADHD are somewhat equivocal. On one hand, there is evidence to suggest that children with ADHD may experience increased pain intensity. Compared with other children, children with ADHD have been shown to have increased tactile defensiveness, which is a tendency to react negatively or with alarm to a sensory input that is generally considered harmless or nonirritating $(4,5)$. This may result in a lower pain threshold and increased response to pain. In addition, 
Table 2 Postoperative pain outcomes

\begin{tabular}{|c|c|c|c|}
\hline & $\begin{array}{l}\text { No ADHD } \\
(n=122)\end{array}$ & $\begin{array}{l}\text { ADHD } \\
(n=119)\end{array}$ & Mean difference $(95 \% \mathrm{Cl})$ \\
\hline Highest NRS pain score PACU & $3.21 \pm 3.0$ & $3.63 \pm 3.4$ & $0.42(-0.4,1.2)$ \\
\hline NRS Pain score at PACU DC & $1.44 \pm 2.0$ & $1.25 \pm 2.0$ & $-0.19(-0.7,0.3)$ \\
\hline Parent preanalgesic pain score & $4.12 \pm 2.6$ & $4.67 \pm 2.9$ & $0.55(-0.2,1.3)$ \\
\hline Child preanalgesic pain score & $4.0 \pm 2.8$ & $4.55 \pm 2.9$ & $0.54(-0.2,1.3)$ \\
\hline $\begin{array}{l}\text { Parent global estimate of } \\
\text { pain since DC }\end{array}$ & $2.8 \pm 1.9$ & $3.3 \pm 2.5$ & $0.48(-0.1,1.1)$ \\
\hline $\begin{array}{l}\text { Perioperative oral morphine } \\
\text { equivalents }\left(\mathrm{mg} \cdot \mathrm{kg}^{-1}\right)\end{array}$ & $0.14 \pm 0.1$ & $0.15 \pm 0.12$ & $0.004(-0.02,0.03)$ \\
\hline $\begin{array}{l}\text { Oral morphine equivalents } \\
\left(\mathrm{mg}^{\prime} \mathrm{kg}^{-1}\right) \text { POD } 1\end{array}$ & $0.08 \pm 0.1$ & $0.12 \pm 0.3$ & $0.04(-0.02,0.09)$ \\
\hline Acetaminophen $\left(\mathrm{mg} \cdot \mathrm{kg}^{-1}\right)$ POD 1 & $17.3 \pm 22.5$ & $15.9 \pm 17.1$ & $-1.3(-6.5,3.9)$ \\
\hline Ibuprofen $\left(\mathrm{mg} \cdot \mathrm{kg}^{-1}\right)$ POD 1 & $2.0 \pm 5.3$ & $3.6 \pm 8.8$ & $1.6(-0.3,3.5)$ \\
\hline Days to normal activity & $3.8 \pm 3.0$ & $4.9 \pm 3.8^{*}$ & $1.1(0.3,1.9)$ \\
\hline Postoperative nausea/vomiting & $19(15.6)$ & $24(20.2)$ & \\
\hline
\end{tabular}

Data are described as mean \pm SD or $n(\%)$. ADHD, attention-deficit hyperactivity disorder; PACU, postanesthesia care unit; DC, discharge; $\mathrm{POD}$, postoperative day, $\mathrm{Cl}$, confidence interval. ${ }^{*} P<0.05$ vs no ADHD.

Table 3 Pain outcomes for children treated with ADHD medications compared with those not treated, and those without ADHD

\begin{tabular}{lcll}
\hline & $\begin{array}{l}\text { No ADHD } \\
(n=122)\end{array}$ & $\begin{array}{l}\text { ADHD not medically } \\
\text { managed }\left(n=25^{*}\right)\end{array}$ & $\begin{array}{l}\text { ADHD on current } \\
\text { medications }\left(n=91^{*}\right)\end{array}$ \\
\hline $\begin{array}{l}\text { Highest pain score in PACU } \\
\text { Pain score at PACU DC }\end{array}$ & $\begin{array}{l}3.18 \pm 3.0 \\
1.43 \pm 2.0\end{array}$ & $\begin{array}{l}2.80 \pm 2.93 \\
0.72 \pm 1.2\end{array}$ & $\begin{array}{l}3.83 \pm 3.44 \\
1.44 \pm 2.1\end{array}$ \\
$\begin{array}{l}\text { Parent-reported preanalgesic } \\
\text { pain score }\end{array}$ & $4.13 \pm 2.6$ & $3.90 \pm 2.9$ & $4.83 \pm 2.9$ \\
$\begin{array}{l}\text { Child-reported preanalgesic } \\
\text { pain score }\end{array}$ & $4.03 \pm 2.8$ & $4.25 \pm 2.9$ & $4.58 \pm 3.0$ \\
$\begin{array}{l}\text { Parent global estimate of } \\
\text { pain since DC }\end{array}$ & $2.83 \pm 1.9$ & $2.86 \pm 2.2$ & $3.45 \pm 2.5$ \\
$\begin{array}{l}\text { Oral morphine equivalents } \\
\left.\text { (mg.kg }{ }^{-1}\right) \text { POD 1 }\end{array}$ & $0.080 \pm 0.11$ & $0.073 \pm 0.11$ & $0.130 \pm 0.31$ \\
\begin{tabular}{l} 
Days to normal activity \\
\hline
\end{tabular} & $3.8 \pm 3.0$ & $4.3 \pm 2.5$ & $5.0 \pm 3.2$ \\
\hline
\end{tabular}

ADHD, attention-deficit hyperactivity disorder; DC, discharge; $P O D$, postoperative day; PACU, postanesthesia care unit.

*Three patients with ADHD with current medication use unknown. the brains of patients with ADHD show a dysfunction in the number of epinephrine receptors in the locus coeruleus, the portion of the brain involved in the descending pain system, which suppresses pain (6,7). The subsequent locus coeruleus hypoactivity experienced by patients with ADHD leads to decreased attention, sleep difficulties, and potential differences in their responses to pain (8). Finally, a study by Kariyawasam et al. (9) examined whether children with ADHD may have hypoactive HPA axes. These authors found that children with ADHD and concurrent oppositional defiant disorder who were not on stimulant medications had reduced salivary cortisol levels, which suggests HPA axis hypoactivity. This is of interest in the perioperative period as corticotropin-releasing factor, a component of the HPA axis that can act on the neuraxis to produce analgesia
(10), may be lower in children with ADHD, possibly leading to increased pain intensity.

On the other hand, there are data to support the concept that children with ADHD may have a decrease in pain experience. High-resolution MRI imaging has compared the brains of children with and without ADHD, and found that children with ADHD have decreased brain size in the inferior portions of the dorsal prefrontal cortices bilaterally. These are centers for executive function, including attention and behavioral inhibition (11) and thus may affect the emotional and cognitive aspects of pain. Also, functional MRI scans during anticipation and administration of noxious stimuli show hypoactive areas of the anterior cingulated cognitive division in the brains of children with ADHD compared with those without ADHD, which may equate with 
decreased influence of the emotional and cognitive aspects of pain leading to a decreased pain experience in children with ADHD (12).

However, in our study, we found no statistically significant difference in pain scores or analgesic use in children with and without ADHD, regardless of whether or not they were currently medically managed. Importantly, our study was powered to detect at least a 1-point difference in NRS scores, which was previously determined to be the average clinically relevant difference in healthy children.

As a secondary outcome, we examined the relationship between time to return to normal activity, as rated by parent observation, in children with and without ADHD. Results revealed a statistically significant difference in time to return to normal activity in children with ADHD. A prior study by Tait et al. (2). showed an increase in maladaptive behaviors in children with ADHD following surgery and anesthesia, which appears consistent with the longer time to return to normal activity observed in this study.

A few comments are worth mentioning regarding the limitations of this study. First, overall pain scores were low in this study, and smaller differences may be considered relevant to children when pain intensity is low, just as bigger differences have been deemed important when pain intensity is high (16). However, a sample of at least 492 children per group would be needed to demonstrate a 0.5 -point difference in pain intensity between groups. Second, due to the design of the study, the anesthetic regimen used was not standardized. However, the groups were similar regarding intraoperative use of analgesics and local/regional anesthetics. Third, the population studied was limited to children aged 7-17 having outpatient or overnight stay procedures, so the ability to generalize findings to other groups may be limited. Finally, although it was not possible to blind all caregiv- ers to the ADHD status of the subjects, the majority of outcome data were recorded by individuals who were unaware that we were specifically studying children with ADHD and who had no vested interests in the outcomes of the study.

We hypothesized, based on the current understanding of the uniqueness of brain structure and function in children with ADHD as well as an increase in tactile defensiveness, that these children would have a difference in perioperative pain experience compared with children without ADHD, as measured by NRS pain scores and analgesic requirements. On the basis of the data obtained in this sample, we reject our hypothesis that there is a difference. However, in agreement with previously published literature, this research does suggest that we can advise patients and families that children with ADHD may take longer to return to baseline activity following surgery and anesthesia compared with children without ADHD.

\section{Funding}

Supported by the Department of Anesthesiology, University of Michigan Health System.

\section{Conflict of interest}

This study was approved by the University of Michigan's Institutional Review Board. There are no conflicts of interest to report.

\section{Acknowledgments}

The authors are indebted to Elsa Pechlivanidis, B.S., B.S.N., and Sarah Zyzanski, B.S. for help with subject recruitment and data collection.

\section{References}

1 Polanczyk G, Silva de Lima M, Horta BL et al. The worldwide prevalence of ADHD: a systematic review and metaregression analysis. Am J Psychiatry 2007; 164: 942-948.

2 Forsyth I, Bergesio R, Chambers N. Editorial: attention-deficit hyperactivity disorder and anesthesia. Pediatr Anesth 2006; 16 371-373.

3 Tait AR, Voepel-Lewis T, Burke C et al. Anesthesia induction, emergence, and postoperative behaviors in children with attention-deficit/hyperactivity disorders. Pediatr Anesth 2010; 20: 323-329.

4 Broring T, Rommelse N, Sergeant J et al. Sex differences in tactile defensive- ness in children with ADHD and their siblings. Dev Med Child Neurol 2008; 50: 129-133.

5 Parush S, Sohmer H, Steinberg A et al. Somatosensory functioning in children with attention deficit hyperactivity disorder. Dev Med Child Neurol 1997; 39: 464-468.

6 Makha S, McMillan J, Iggo A. Pathways mediating descending control of the spinal nociceptive transmission from the nuclei locus coeruleus (LC) and raphe magnus (NRM) in the cat. Exp Brain Res 1986; 61: 597-606.

7 Willis W, Westlund K. Neuroanatomy of the pain system and of the pathways that modulate pain. J Clin Neurophysiol 1997; 14: 2-31.
8 Mefford I, Potter W. A neuroanatomical and biochemical basis for attention deficit disorder with hyperactivity in children: a defect in tonic adrenaline mediated inhibition of locus coeruleus stimulation. Med Hypotheses 1989; 29: 33-42.

9 Kariyawasam S, Zaw F, Handley S. Reduced salivary cortisol in children with comorbid attention deficit hyperactivity disorder and oppositional defiant disorder. Neuro Endocrinol Lett 2002; 23: 45-48.

10 Lariviere W, Melzack R. The role of corticotropin-releasing factor in pain and analgesia. Pain 2000; 84: 1-12.

11 Sowell E, Thompson P, Welcome S et al. Cortical abnormalities in children and ado- 
lescents with attention-deficit hyperactivity disorder. Lancet 2003; 362: 1699-1707.

12 Bush G, Frazier J, Rauch S et al. Anterior cingulated cortex dysfunction in attentiondeficit/hyperactivity disorder revealed by fMRI and the Counting Stroop. Biol Psychiatry 1999; 45: 1542-1552.

13 von Baeyer CL, Spagrud LJ, McCormick JC et al. Three new datasets supporting use of the Numerical Rating Scale (NRS-11) for children's self-reports of pain intensity. Pain 2009; 143: 223-227.

14 Voepel-Lewis T, Burke C, Jeffreys $\mathrm{N}$ et al. Do 0-10 numeric rating scores translate into clinically meaningful pain measures for children? Anesth Analg 2011; 112: 415-421.

15 Malviya S, Voepel-Lewis T, Chiravuri SD et al. Does an objective system-based approach improve assessment of perioperative risk in children? A preliminary evaluation of 'NARCO'. Br J Anaesth 2011; 106: 352-358

16 Voepel-Lewis T. Pain assessment and decision-making: have we missed the mark? Pediat Pain Lett 2011; 13: 1-6. 